\title{
DIFERENCIAÇÃO COMPLEXA E AS CONDIÇÕES DE CAUCHY-RIEMANN
}

Pâmela Catarina de Sousa Brandão ${ }^{1}$, Fernando Pereira Sousa ${ }^{2}$

${ }^{1}$ Aluna do Curso de Matemática - CPTL/UFMS, bolsista do Grupo PET Conexões de Saberes - Matemática/CPTL/UFMS;

${ }^{2}$ Professor do Curso de Matemática - CPTL/UFMS. E-mail: pamela31 brandao@hotmail.com

\section{RESUMO}

Neste trabalho estudamos alguns conceitos de Funções Complexas, comparamos os resultados obtidos com o caso de funções reais e funções de várias variáveis. O principal resultado do nosso trabalho foi demonstrar quais condições são necessárias e suficientes para que uma função complexa seja diferenciável, tais condições são conhecidas como condições de Cauchy-Riemann. Para demonstrarmos esse teorema fizemos a apropriação dos conceitos de função(funções exponenciais e trigonométricas), limite e derivada no plano complexo, bem como exemplificações. Palavras Chave: Funções Complexas, Exponencial, Cauchy-Riemann, Derivadas Parciais, Diferenciação.

\section{INTRODUÇÃo}

O presente trabalho aborda assuntos de funções de uma variável complexa, mais concretamente às funções exponenciais e trigonométricas, limite e diferenciação de funções complexas, no qual mostramos que para que uma função complexa $f$ seja diferenciável é necessário que as partes real e imaginária de $f$ satisfaçam um certo sistema de equações diferenciais parciais, conhecidas como equações de Cauchy-Riemann.

O objetivo deste trabalho é apresentar o estudo do conceito de função de uma variável complexa, dando ênfase especial ao conceito de limite e derivação. Discutimos alguns dos resultados mais importantes da teoria de funções complexas, em particular, demonstramos um resultado importante que nos dá condições necessárias para que uma função seja diferenciável, acrescentamos um teorema que com uma certa hipótese adicional sobre a função, a validade das equações de Cauchy-Riemann é uma condição suficiente para a diferenciabilidade. Otrabalho é finalizado com um exemplo que envolve esses dois resultados.

\section{METODOLOGIA}

O trabalho realizado é resultado de um estudo detalhado, desenvolvido através de discussões do tema com o orientador e apresentações de seminários como parte das atividades do programa PET Conexões de Saberes Matemática - UFMS/CPTL e do Trabalho de Conclusão de 
Curso. Para o desenvolvimento desse trabalho, fizemos o estudo detalhado dos conceitos, bem como de exemplos, relacionados com a teoria desenvolvida.

\section{RESULTADOS}

Sejam $A$ e $B$ conjuntos arbitrários. Uma aplicação $f$ de $A$ em $B$ é uma regra de correspondência que associa a cada elemento $a$ de $A$ um único elemento $f(a)$ de $B$, chamado valor de $f$ em a. Usaremos a notação

$$
\begin{gathered}
f: A \rightarrow B \\
a \in A \mapsto f(a) \in B
\end{gathered}
$$

para indicar que $f$ é uma tal função. O conjunto $A$ é chamado o domínio de $f$ e o conjunto $B$ é chamado o contradomínio de $f$. O conjunto $f(A)$ é chamado a imagem de $f$. Quando $f(A)=B$, dizemos que $f$ é sobrejetiva. Se $f\left(a_{1}\right) \neq f\left(a_{2}\right)$ sempre que $a_{1} \neq a_{2}$, com $a_{1}, a_{2} \in A$ dizemos que $f$ é injetiva. E por fim, se $f$ é injetiva e sobrejetiva dizemos que ela é bijetiva.

Podemos escrever uma função $f: A \rightarrow \mathbb{C}$ em termos de sua parte real e de sua parte imaginária, ou seja,

$$
f=u+i v o n d e ~ u(z)=\operatorname{Re}[f(z)] \text { e } v(z)=\operatorname{Im}[f(z)], z \in A .
$$

Além disso, ue $v$ são funções reais em $A$. Se escrevermos $z=(x, y) \operatorname{com} x, y \in \mathbb{R}$, teremos que $u$ e $v$ são funções de duas variáveis reais: $u(z)=u(x, y)$ e $v(z)=v(x, y)$.

Dentre todas as funções complexas, estudaremos as funções exponenciais e as trigonométricas.

A função exponencial é a função exp: $\mathbb{C} \rightarrow \mathbb{C}$ dada por

$$
\exp z=e^{z}=e^{x+i y}=e^{x}(\cos y+i \operatorname{sen} y) \text {. }
$$

Como $\left|e^{z}\right|=\left|e^{x+i y}\right|=\left|e^{x} \cdot e^{i y}\right|=\left|e^{x}(\cos y+i \operatorname{sen} y)\right|=\left|e^{x}\right|=e^{x}$, para todo $z=x+i y \in \mathbb{C}$, temos que $\exp z \neq 0$ para todo $z \in \mathbb{C}$.Além disso, é importante dizer que a função exponencial é uma função periódica de período $2 \pi i$, ou seja,exp $(z+2 \pi i)=\exp z$, para todo $z \in \mathbb{C}$. De fato,

$$
\exp (z+2 \pi i)=e^{z+2 \pi i}=e^{z} \cdot e^{2 \pi i}=e^{z}(\cos 2 \pi+i \operatorname{sen} 2 \pi)=e^{z}(1+i 0)=e^{z} .
$$

Paray $\epsilon \mathbb{R}$, temos que $e^{i y}=\cos y+i$ sen $y$ e $e^{-i y}=\cos y-i$ sen $y$, adicionando os dois membros dessas equações obtemos $\cos y=\frac{e^{i y}+e^{-i y}}{2}$, e subtraindo os dois membrostemos sen $y=\frac{e^{i y}-e^{-i y}}{2 i}$. Sendo assim, definimos a função seno e a função cosseno de uma variável complexa por 


$$
\operatorname{sen} z=\frac{e^{i z}-e^{-i z}}{2 i} \operatorname{e} \cos z=\frac{e^{i z}+e^{-i z}}{2} .
$$

Sejam $f: A \rightarrow \mathbb{C}$ uma função e $z_{0}$ um ponto de $A$. Dizemos que a função $f$ tem o número complexo $w_{0}$ como seu limite em $z_{0}$ se, para todo $\varepsilon>0$, existe $\delta=\delta\left(\varepsilon, z_{0}\right)>0$ tal que

$$
\left|f(z)-w_{0}\right|<\varepsilon \text { sempre que } z \in A \text { e } 0<\left|z-z_{0}\right|<\delta .
$$

(i) Observe que se existe um número complexo $w_{0}$ que satisfaça a definição acima, então ele é único. Escrevemos $\lim _{z \rightarrow z_{0}} f(z)=w_{0}$ ou $f(z) \rightarrow w_{0}$ quando $z \rightarrow z_{0}$ para expressar que o limite de $f$ no ponto $z_{0}$ existe e vale $w_{0}$.

A seguir, algumas propriedades de limites de funções complexas, as demais são análogas ao caso das funções reais.

Suponhamos que a função $f: A \rightarrow \mathbb{C}$ satisfaze $f(z) \rightarrow w_{0}$ quando $z \rightarrow z_{0}$, onde $z_{0}$ é um ponto de $A$. Então, quando $z \rightarrow z_{0}$, temos que:

(ii) $\overline{f(z)}=\overline{w_{0}}$,

(iii) $|f(z)| \rightarrow\left|w_{0}\right|$.

Exemplo: $\lim _{z \rightarrow 4 i} \frac{\mathrm{z}^{3}-8 \mathrm{iz}^{2}-4 \mathrm{z}}{(\mathrm{z}-4 \mathrm{i})^{2}}=\lim _{z \rightarrow 4 i} \frac{\mathrm{z}(\mathrm{z}-4 \mathrm{i})^{2}}{(\mathrm{z}-4 \mathrm{i})^{2}}=\lim _{z \rightarrow 4 i} z=4 i$

Agora, definimos os conceitos de diferenciabilidade e derivada de funções complexas de uma variável complexa, apresentamos alguns exemplos e propriedades, dentre elas, a regra da cadeia.

A derivada de uma função $f$, complexa, no ponto $z_{0}$, denotada por $f^{\prime}\left(z_{0}\right)$, é definida por $f^{\prime}\left(z_{0}\right)=\lim _{\Delta \rightarrow z_{0}} \frac{f\left(z_{0}+\Delta_{z}\right)-f\left(z_{0}\right)}{\Delta_{z}}$, desde que esse limite exista. Se este for o caso, $f$ é dita diferenciável em $z_{0}$. Se considerarmos $\Delta z=z-z_{0}$, temos $z=\Delta z+z_{0}$, sendo assim,

$$
f^{\prime}\left(z_{0}\right)=\lim _{z \rightarrow z_{0}} \frac{f(z)-f\left(z_{0}\right)}{z-z_{0}}
$$

As propriedades de limites de funções complexas são demonstradas de modo análogo ao caso real.

A partir daqui deixaremos de ter a impressão que o "cálculo diferencial complexo" é completamente análogo a "cálculo diferencial real". As diferenças entre essas duas teorias são profundas, uma primeira diferença aparece numa proposição, que diz: para que uma função $f: A \rightarrow \mathbb{C}$ seja diferenciável em um ponto $z_{0}$ é necessário que haja uma certa compatibilidade entre as derivadas parciais das partes real e imaginária de $f$ nesse ponto, que é dada pelas equações de Cauchy-Riemann. 
Para o estudo dessas condições dizemos que uma função fé Holomorfa ou Analítica num domínio $D$ se $f$ é definida e diferenciável em todos os pontos de $D$. A função $f$ é dita analítica num ponto $z_{0}$ em $D$ se $f$ for analítica numa vizinhança de $z_{0}$.

Teorema:Seja $f(z)=u(x, y)+i v(x, y)$ uma função definida e contínua em alguma vizinhança do ponto $z=x+i y$ e diferenciável em $z$. Então, as derivadas parciais de primeira ordem de $u$ e de $v$ existem e satisfazem às equações

$$
\frac{\partial}{\partial x} \mathrm{u}(\mathrm{x}, \mathrm{y})=\frac{\partial}{\partial \mathrm{y}} \mathrm{v}(\mathrm{x}, \mathrm{y}) \text { e } \frac{\partial}{\partial y} \mathrm{u}(\mathrm{x}, \mathrm{y})=-\frac{\partial}{\partial \mathrm{x}} \mathrm{v}(\mathrm{x}, \mathrm{y})
$$

conhecidas como as condições de Cauchy-Riemann. Então, se $f$ é analítica num domínio $D$,suas derivadas parciais existem e satisfazem as equações acima em todos os pontos do domínio $D$.

Dem: Sabendo da definição de limite que podemos usar qualquer direção para fazer $z$ tender a $z_{0}$ vamos escolher dois caminhos, o primeiro, o paralelo ao eixo real, ou seja, $\operatorname{com} \Delta_{y}=0$, e o segundo, paralelo ao eixo imaginário, de onde $\Delta_{x}=0$, conforme na figura abaixo.

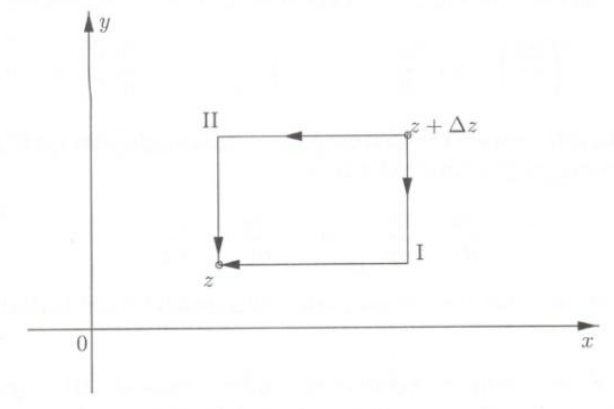

Para função complexa $f(x, y)=u(x, y)+i v(x, y)$ temos

$$
\left(\frac{d f}{d z}\right)_{z=z_{0}}=\lim _{\substack{\Delta_{x} \rightarrow 0 \\ \Delta_{y} \rightarrow 0}}\left(\frac{u\left(x_{0}+\Delta_{x}, y_{0}+\Delta_{y}\right)-u\left(x_{0}, y_{0}\right)}{\Delta_{x}+i \Delta_{y}}+\frac{i\left[v\left(x_{0}+\Delta_{x}, y_{0}+\Delta_{y}\right)-v\left(x_{0}, y_{0}\right)\right]}{\Delta_{x}+i \Delta_{y}}\right) .
$$

Como este limite deve existir e ser o mesmo por qualquer um dos caminhos, então, para $\Delta_{y}=0$, temos

$$
\left(\frac{d f}{d z}\right)_{z=z_{0}}=\lim _{\Delta_{x} \rightarrow 0} \frac{u\left(x_{0}+\Delta_{x}, y_{0}\right)-u\left(x_{0}, y_{0}\right)}{\Delta_{x}}+i \lim _{\Delta_{x} \rightarrow 0} \frac{v\left(x_{0}+\Delta_{x}, y_{0}\right)-v\left(x_{0}, y_{0}\right)}{\Delta_{x}}
$$

Esses quocientes são a definição de derivada parcial, logo

$$
\left(\frac{d f}{d z}\right)_{z=z_{0}}=\left(\frac{\partial u}{\partial x}\right)_{x_{0}, y_{0}}+i\left(\frac{\partial v}{\partial x}\right)_{x_{0}, y_{0}}
$$

Agora, para o segundo caminho, $\Delta_{x}=0$, temos de maneira análoga, que

$$
\left(\frac{d f}{d z}\right)_{z=z_{0}}=\lim _{\Delta_{y} \rightarrow 0} \frac{v\left(x_{0}, y_{0}\right)-v\left(x_{0}, y_{0}+\Delta_{y}\right)}{\Delta_{y}}-i \lim _{\Delta_{y} \rightarrow 0} \frac{u\left(x_{0}, y_{0}+\Delta_{y}\right)-u\left(x_{0}, y_{0}\right)}{\Delta_{y}} \text {. }
$$

Ou seja, 


$$
\left(\frac{d f}{d z}\right)_{z=z_{0}}=\left(\frac{\partial v}{\partial y}\right)_{\left(x_{0}, y_{0}\right)}-i\left(\frac{\partial u}{\partial y}\right)_{\left(x_{0}, y_{0}\right)}
$$

Sendo assim,

$$
\left(\frac{\partial u}{\partial x}\right)_{\left(x_{0}, y_{0}\right)}+i\left(\frac{\partial v}{\partial x}\right)_{\left(x_{0}, y_{0}\right)}=\left(\frac{\partial v}{\partial y}\right)_{\left(x_{0}, y_{0}\right)}-i\left(\frac{\partial u}{\partial y}\right)_{\left(x_{0}, y_{0}\right)},
$$

ou seja, igualando as partes reais e as partes imaginárias, segue que $\frac{\partial u}{\partial x}=\frac{\partial \mathrm{v}}{\partial \mathrm{y}} \mathrm{e} \frac{\partial u}{\partial y}=-\frac{\partial \mathrm{v}}{\partial \mathrm{x}}$.

Por exemplo, se considerarmos a função $f(z)=\bar{z}$, temos que

$$
u(x, y)=x \text { e } v(x, y)=-y \text { quando } z=x+i y \in \mathbb{C} .
$$

Temos que $\frac{\partial u}{\partial x}=1, \frac{\partial v}{\partial y}=-1, \frac{\partial u}{\partial y}=0 e \frac{\partial v}{\partial x}=0$, ou seja, $\frac{\partial u}{\partial x} \neq \frac{\partial v}{\partial y} \mathrm{e} \frac{\partial u}{\partial y}=\frac{\partial v}{\partial x}$ para todo $z \in \mathbb{C}$. Logo, $f$ não é diferenciável.

O fato das derivadas parciais de $f$ satisfazerem as condições de Cauchy-Riemann é uma condição necessária para que $f$ seja diferenciável num ponto $z_{0}$, porém não é suficiente. 0 seguinte teorema nos da condições que são suficientes para diferenciabilidade de $f$.

Teorema: Suponhamos que uma função $f=u+i v$ esta definida em um conjunto aberto $A$ de $\mathbb{C}$ e que as derivadas parciais $\frac{\partial u}{\partial x}, \frac{\partial u}{\partial y}, \frac{\partial v}{\partial x} e \frac{\partial v}{\partial y}$ existem em todo ponto de $A$. Se cada uma dessas derivadas parciais é contínua em um ponto $z_{0}$ de $A$ e se as equações de Cauchy-Riemann são satisfeitas por $u$ e $v$ em $z_{0}$, então $f$ é diferenciável em $z_{0}$.

Exemplo: A função $f(x)=e^{z}=e^{x}(\cos y+i \operatorname{sen} y)=e^{x} \cos y+i e^{x} \operatorname{sen} y \quad$ é diferenciável, pois temos $u(x, y)=e^{x} \cos y$ e $v(x, y)=e^{x} \operatorname{sen} y$, onde $\frac{\partial u}{\partial x}=\frac{\partial \mathrm{v}}{\partial \mathrm{y}}=e^{x} \cos y$ e $\frac{\partial u}{\partial y}=-\frac{\partial \mathrm{v}}{\partial \mathrm{x}}=e^{x} \operatorname{sen} y$, para todo $z \in \mathbb{C}$, como as derivadas parciais são contínuas temos que $f$ é diferenciável em todos os pontos de $\mathbb{C}$.

\section{DISCUSSÃO}

As condições de Cauchy-Riemann nos ajuda a entender a diferença entre o estudo de funções complexas e funções reais, principalmente quando estudamos a diferenciabilidade de funções complexas.

As técnicas utilizadas no trabalho permitem o desenvolvimento do estudo de integração complexa, pretendemos estudar as aplicações da integral de Cauchy, Séries de Taylor e Séries de Laurent, que constituem a continuidade natural da investigação do trabalho. 


\section{CONCLUSÕES}

Neste trabalho abordamos alguns tópicos de funções de uma variável complexacom maior enfoque em diferenciação. No estudo de funções diferenciáveis vimos que uma função é analítica se satisfaz as condições de Cauchy-Riemann e suas derivadas parciais são contínuas, este fato interessante, nos deixa claro a diferença entre funções complexas e reais.

Finalizamos o trabalho enunciando e demonstrando o teorema das Condições de CauchyRiemann, cujo resultado fornece condições necessárias para que uma função de uma variável complexa seja diferenciável, e em seguida enunciamos um teorema que com uma certa hipótese adicional sobre a função, a validade das equações de Cauchy-Riemann é uma condição suficiente para a diferenciabilidade.

O estudo da diferenciabilidade de funções de uma variável complexa permitiu desenvolver um trabalho com conceitos matemáticos que não faz parte da grade curricular do curso de Matemática-Licenciatura da UFMS/CPTL, enriquecendo meus conhecimentos sobre o assunto abordado.

\section{REFERÊNCIAS}

1. FERNANDES, C.S.; BERNARDES Jr, N.C.; Introdução ás Funções de uma Variável Complexa.2.ed. Rio de Janeiro: SBM, 2008.

2. LINS NETO, A.;Funções de uma Variável Complexa.2.ed.Rio de Janeiro: IMPA, 2008.

3. OLIVEIRA, E.C.; RODRIGUES Jr, E.C.;Funções Analíticas com Aplicações.1.ed.São Paulo: Livraria da Física, 2006. 